\title{
EXERCISE CAPACITY AND FUNCTIONAL STATUS IN LIVER TRANSPLANT CANDIDATES: IDENTIFYING PERCEIVED DETERMINANTS AND CONSEQUENCES
}

\author{
Tuba Ergene ${ }^{1}$, Didem Karadibak ${ }^{2}$, Sule Namli Koc ${ }^{3}$ \\ ${ }^{1}$ Department of Cardiopulmonary Physiotherapy and Rehabilitation, Memorial Atasehir Hospital. \\ 2 Department of Cardiopulmonary Physiotherapy, Dokuz Eylul University, School of Physical Therapy and Rehabilitation. \\ İzmir, Turkey. \\ ${ }^{3}$ Department of Gastroenterology, Memorial Atasehir Hospital.
}

Address for Correspondence: Tuba Ergene, PT, MSc, E-mail: tuba@ergene.net Received: 20.02.2021; Accepted: 30.03.2021; Available Online Date: 27.05.2021

(C) Copyright 2021 by Dokuz Eylül University, Institute of Health Sciences - Available online at https://dergipark.org.tr/en/pub/jbachs

Cite this article as: Ergene T, Karadibak D, Namli-Koc S. Exercise Capacity and Functional Status in Liver Transplant Candidates: Identifying Perceived Determinants and Consequences. J Basic Clin Health Sci 2021; 2: 49-58

\begin{abstract}
Purpose: Exercise capacity is a well known prognostic indicator in many clinical conditions. This study evaluates the clinical and exercise variables most determinant for estimating exercise capacity in liver transplant candidates.

Methods: This prospective cross-sectional study consisted of thirty patients who were scheduled for liver transplantation. Respiratory and peripheral muscle strengths were measured using a mouth pressure device and a hand-held dynamometer, respectively. Physical activity, fatigue, exercise capacity, physical performance, and quality of life of participants were assessed using the International Physical Activity Questionnaire, the Turkish version of the Checklist Individual Strength Questionnaire, the Six-minute Walk Test (6MWT), the 30-sec Sit-to-Stand Test, and the Liver Disease Symptom Index 2.0, respectively. Multivariate analysis was conducted to determine clinical factors predictive of exercise impairment.
\end{abstract}

Results: The participants achieved $62.9 \%$ of the predicted six-minute walk distance (6MWD). 6MWD was strongly correlated with age $(r=-0.678, p<0.001)$, dyspnea before the 6MWT $(r=-0.668, p<0.001)$, and chair stands $(r=0.745, p<0.001)$. A moderate correlation was observed between $6 \mathrm{MWD}$ and maximal inspiratory pressure $(r=0.532, p=0.002)$, maximal expiratory pressure $(r=0.522, p=0.003)$, and shoulder flexion $(r=0.479, p=0.007)$. Age and maximal expiratory pressure were identified as independent determinants of exercise capacity, accounting for $57.4 \%$ of the variance.

Conclusion: Assessing respiratory parameters may have a fundamental role in improving posttransplant outcomes in especially older liver transplant candidates.

Key Words: End-Stage Liver Diseases, Exercise Capacity, Functional Status

\section{INTRODUCTION}

Chronic liver failure, one of the top 20 critical causes of disability-adjusted life years, is a diffuse parenchymal liver disease process characterized by fibrosis and abnormal nodules, representing the final histological change for end-stage liver diseases
(ESLD) (1). The disorders caused by liver failure lead to metabolic alterations and systemic manifestations, including a gradual loss of muscle mass and strength, reduced physical activity level and, consequently, impaired functional status. Regarding respiratory impairment related to chronic hepatopathy, chronic 
ascites has a pressure effect changing the curvature and the length-tension relation of the diaphragm, thus increasing the respiratory muscles' workload, leading breathing difficulties $(2,3)$. All these consequences of the disease negatively influence the daily living activities (ADL) and health-related quality of life (HRQoL) of the liver failure patient population and increase the wait-list mortality both pre and postoperatively, independent of the etiology $(4,5)$. Also, additional complications may occur due to the long waiting period, resulting in worsened functionality. The present study aims 1) to determine whether there is an association between liver disease severity, physical activity level, respiratory and skeletal muscle strength, fatigue perception, exercise capacity, and HRQoL 2) to predict the determinants of exercise capacity in patients awaiting liver transplantation (LT).

\section{METHODS}

\section{Study Design/Participants}

The study was based on cross-sectional analysis. Thirty ambulatory patients scheduled for LT with a clinical diagnosis of ESLD in XXX Hospital between September 2018 through May 2019 were included. The study included patients who agreed to participate, aged $\geq 18$ years, listed for a first $L T$, and can read, write, and understand Turkish. The exclusion criteria were having severe comorbidities (e.g., cardiovascular instability), primary lung diseases (e.g., interstitial lung disease), advanced musculoskeletal and psychological limitations, inability to understand verbal commands, and being a multiorgan transplant recipient and diagnosis of acute liver failure. The DEU Non-Interventional Research Ethics Committee (No: 2017/21-61) approved the study protocol, which was conducted following the principles outlined in the Declaration of Helsinki. After being informed about the research, individuals signed written informed consent regarding participating in the study and publishing their data.

\section{Sample Size}

The sample size was calculated applying data from van Ginneken et al.'s study, in which they evaluated the relationship between physical performance and fatigue and quality of life within a group of liver transplant recipients (6). They reported the difference between the 6-min walk distance of subjects and norm values. A total sample size of 30 participants (15 cases in each group) was calculated using "Epi
Info Statcalc Version 6" with a 95\% confidence interval and $80 \%$ power.

\section{Data collection}

The participants underwent a pre-liver transplant evaluation by the medical team, including physical examination, laboratory tests, radiological imaging, surgery-specific consultations, and were categorized in The American Society of Anesthesiologists (ASA) physical status classification. All physiotherapeutic evaluations were applied by a staff physiotherapist who has a cardiopulmonary speciality. Subjects' sociodemographic characteristics were recorded in a detailed survey.

The disease severity was assessed by the Model for End-Stage Liver Disease (MELD) scoring system, to predict three months of mortality and is considered a valuable tool to predict the functional capacity and respiratory muscle status in LT candidates (2). MELD score objectively stratifies patients on a scale between 6-40 points, with higher values indicating severe disease, and it incorporates three laboratory parameters [serum creatinine and total bilirubin, and international normalized ratio (INR)].

Physical Activity (PA) level was assessed by the Turkish version of the International Physical Activity Questionnaire Short Form (IPAQ-SF), and permission to use the questionnaire was previously obtained (7). It includes seven questions to determine the frequency, intensity, and duration of self-reported participation in PA. IPAQ-SF also questions the length of sitting time ( $\mathrm{min} /$ day). While scoring, first, minutes per week spent in walking, moderateintensity activity, and vigorous-intensity activity are calculated, then the results are multiplied by the activity's corresponding metabolic equivalent (MET) to estimate the total score (MET-min/week). Subjects are categorized as light (L: 3.3 METs), moderate (M: 4.0 METs), and vigorous ( $\mathrm{V}:$ : 8.0 METs).

\section{Muscle Strength; (a) Respiratory muscle strength was evaluated by measuring mouth pressures (Micro MPM; Micro Medical Ltd, England) through maximal inspiratory pressure (MIP) during maximal inspiration following a full expiration and maximal expiratory pressure (MEP) during a maximal expiration following a total inspiration. While testing, subjects used a nose clip. They sealed their lips}


around the mouthpiece to prevent air leakage. The participants were encouraged to repeat the test maneuvers three times, and the highest measured value was recorded as $\mathrm{cmH}_{2} \mathrm{O}$. All obtained values were also expressed as a percentage of predicted (pred) values using equations (8). (b) The peripheral muscle strength (knee extension, shoulder flexion, and abduction) was assessed using a hand-held dynamometer (Power Track Commander II, J-Tech Medical Industries, Salt Lake City, USA). Subjects performed three sustained maximal isometric contractions under the previously defined testing positions, with a 1-min rest period (9). The highest measured value during the preserved maximum tension for $5 \mathrm{~s}$ was recorded in kilograms $(\mathrm{kg})$. The predicted values were calculated individual's equations (9).

Fatigue was evaluated using the Checklist Individual Strength-Turkish version (CIS-T) questionnaire (10) after getting permission to use the material. The 20 item self-report instrument evaluates fatigue perception experienced in the preceding two weeks. The questionnaire contains many aspects of fatigue, as subjective fatigue (8-items), concentration (5items), motivation (4-items), PA (3-items) scored using a seven-point

Likert-type scale (1[Yes] to $7[\mathrm{No}]$ ) in each subgroup. A total score was calculated, where higher scores indicated severe fatigue. The subjects were distinguished using a cutoff score (a CIS total score $>76$ ).

Exercise capacity was measured using the six-minute walk test (6MWT) according to guidelines (11). The participants walked for as long as possible in an enclosed $30 \mathrm{~m}$-corridor after being instructed about the procedure. The vitals, including heart rate and oxygen saturation, were recorded before and after testing. Patients were questioned every minute using the Modified Borg Scale (MBS), previously used in liver recipients, to assess the perceived difficulty in breathing and perceived exertion during the test (12). MBS starts at 0 , where breathing is causing the patient no difficulty and progresses through the score of 10 , where breathing difficulty is maximal. The distance covered in 6-minutes was recorded as the six-minute walk distance (6MWD) (13).

Physical performance was evaluated with 30 -sec Sitto-Stand Test (STST). Subjects were asked to repeat the cycle of standing up fully and sitting back down entirely at their own pace, starting seated in a 45-47 $\mathrm{cm}$ height chair with arms across the chest (14). The number of full stands done in $30 \mathrm{sec}$ was recorded. Two trials with a resting period of $5 \mathrm{~min}$ were conducted; the best score was recorded.

Health-related Quality of Life was assessed using the Turkish version of Liver Disease Symptom Index 2.0 (LDSI 2.0), a disease-specific questionnaire for chronic liver failure, and permission to use the questionnaire was obtained (15). The LDSI 2.0 is an assessment tool that evaluates the symptom severity and its effects on ADL. In the content of 24 items, Appendix I offers 18 . The nine are about itchiness, regional abdominal pain, joint discomfort, insomnia, family concern, poor appetite, depressive symptoms, fear of developing complications, and jaundice; and the other nine measure the impact of all those symptoms on their daily life. The influence of itchiness on both ADL and sleepiness is also measured. Appendix 2 contains difficulties in memory, personality changes, financial status, time use, decreased libido, and sexual life. Here, patients' previous week are questioned, and they are all answered as "none" to "always" rated between 0-4. Higher scores reveal a worsened HRQoL.

\section{Statistical Analysis}

The data set analysis was conducted using SPSS Statistics V22.0 Software (IBM Corp, New York, USA). Descriptive statistics available for categorical variables are frequencies/percentages. Continuous variables were generated with a mean and a standard deviation based on normal distribution; when the shape of the distribution is not a normal distribution, the data were presented as a median and interquartile range. Visual (histograms/probability plots) and analytical methods (KolmogorovSimirnov/Shapiro-Wilk's test) were used to determine whether or not the variables are normally distributed. The correlation coefficients and their significance were determined using the Pearson/Spearman test where appropriate. Variables significantly associated with 6MWD in the univariate analysis were included in multiple stepwise backward regression modeling as potential factors predicting exercise capacity. The model fit was assessed using appropriate residual and goodness-of-fit statistics. The correlation coefficient ranges were determined 
Table 1: Demographic and Clinical Characteristics of Liver Transplant Candidates.

\begin{tabular}{|c|c|c|}
\hline Patients, $n=30$ & \multicolumn{2}{|c|}{ Mean $\pm S D$} \\
\hline Age (years) & \multicolumn{2}{|c|}{$53.20 \pm 12.40$} \\
\hline $\mathrm{BMI}\left(\mathrm{kg} / \mathrm{m}^{2}\right)$ & \multicolumn{2}{|c|}{$26.80 \pm 4.43$} \\
\hline \multirow[t]{2}{*}{ MELD Score } & \multicolumn{2}{|c|}{$17.70 \pm 6.04$} \\
\hline & $\mathbf{n}$ & Percent (\%) \\
\hline \multicolumn{3}{|l|}{ Gender } \\
\hline Female & 10 & 33.3 \\
\hline \multicolumn{3}{|l|}{ Smoking Habit } \\
\hline Present Smoker & 10 & 33.3 \\
\hline Non-smoker & 16 & 53.3 \\
\hline Ex-smoker & 4 & 13.3 \\
\hline \multicolumn{3}{|l|}{ Alcohol Use } \\
\hline Yes & 8 & 26.7 \\
\hline No & 19 & 63.3 \\
\hline Ex-user & 3 & 10 \\
\hline \multicolumn{3}{|l|}{ Exercise Habit } \\
\hline Yes & 11 & 36.7 \\
\hline \multicolumn{3}{|l|}{ Operational History } \\
\hline Yes & 14 & 46.7 \\
\hline \multicolumn{3}{|l|}{ Etiology } \\
\hline $\mathrm{HCV}$ & 1 & 3.3 \\
\hline HBV & 4 & 13.3 \\
\hline Alpha-1 antitrypsin deficiency & 1 & 3.3 \\
\hline Hepatosteatosis & 1 & 3.3 \\
\hline $\mathrm{HCC}$ & 2 & 6.7 \\
\hline $\mathrm{HCC}+\mathrm{HBV}$ & 3 & 10 \\
\hline Cryptogenic & 9 & 30 \\
\hline Ethanol & 7 & 23.3 \\
\hline Autoimmune Hepatitis & 2 & 6.7 \\
\hline \multicolumn{3}{|l|}{ ASA Class } \\
\hline III & 18 & 40 \\
\hline IV & 12 & 60 \\
\hline \multicolumn{3}{|l|}{ Time Since the Diagnosis of CLF } \\
\hline One Year and Above & 22 & 73.3 \\
\hline \multicolumn{3}{|l|}{ Ascites } \\
\hline Yes & 16 & 53.3 \\
\hline \multicolumn{3}{|l|}{ Other Complications } \\
\hline Confusion+Encephalopathy & 2 & 6.7 \\
\hline Esophageal Variceal Bleeding & 5 & 16.7 \\
\hline Icterus & 4 & 13.3 \\
\hline Itchy skin & 1 & 3.3 \\
\hline Fatigue & 17 & 56.7 \\
\hline Nausea & 1 & 3.33 \\
\hline
\end{tabular}

SD: standard deviation, BMI: Body Mass Index, HT: Hypertension, DM: Diabetes mellitus. MELD: Model for End-Stage Liver Disease, HCV: Hepatitis C virus, HBV: Hepatitis B virus, HCC: Hepatocellular carcinoma, ASA: The American Society of Anesthesiologists, CLF: Chronic Liver Failure. 


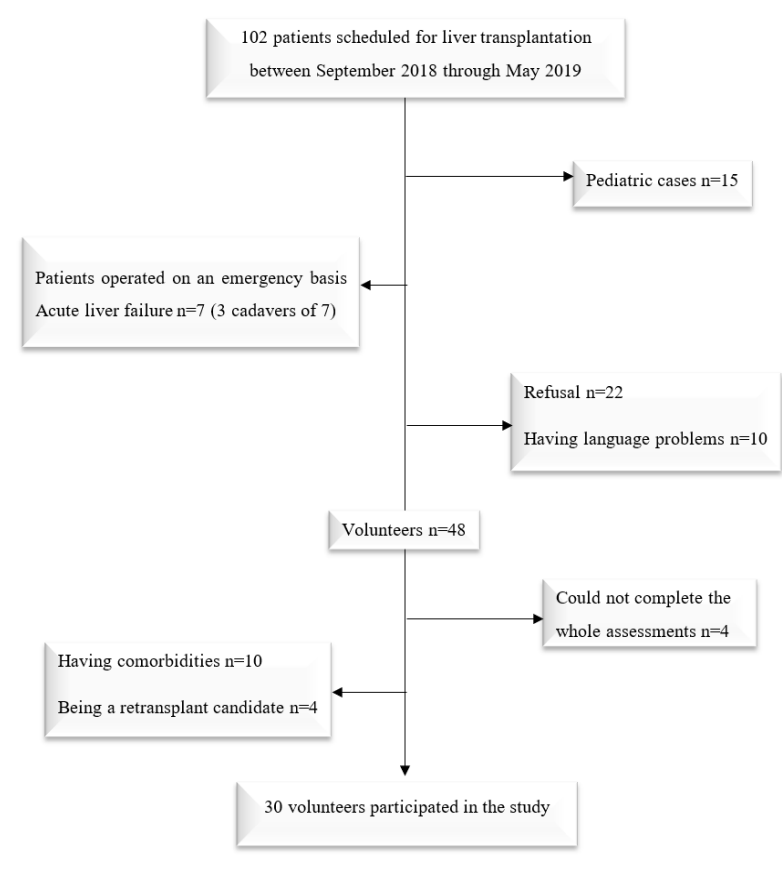

Figure 1. Flowchart of study participants included in the present analysis.

as very weak to very strong (16). A probability of typeI error was set to $5 \%(p<0.05)$.

\section{RESULTS}

Data on 30 liver transplant candidates were analyzed. The flowchart of the participants is given in Figure 1. Participants (53.20 \pm 12.40 years; range: $21-71)$ were tested at an average of $2.1 \pm 3.2$ days pre-operatively. The mean body mass index (BMI) value in our population indicated a mild overweight. The demographic and clinical characteristics of the study sample are given in Table 1. According to the MELD scoring system, 11 patients $(36.7 \%)$ had a score $<15$. The most common etiological causes of liver failure among subjects were cirrhosis due to cryptogenic $(30 \%)$, ethanol $(23.3 \%)$, viral hepatitis, and hepatocellular carcinoma $(\mathrm{HCC})$, or both $(n=10$; $33.3 \%)$.

Clinical evaluations are shown in Table 2. Measured respiratory and peripheral muscle strength were lower compared with the normative reference values. When assessed by IPAQ categorical classification, $63.3 \%$ of patients were inactive. The fatigue perception was seen with the higher rates $(70 \%)$, exceeding the cutoff score. The mean achieved
6MWD was below the percentage of the predicted normal values.

Table 3 presents the correlations between 6MWD and clinical variables. 6MWD was negatively and strongly correlated with age $(r=-0.678, p<0.001)$ and dyspnea rated at the beginning of the test $(r=-0.668$, $\mathrm{p}<0.001$ ) and had a strong positive correlation with STST score $(r=0.745, p<0.001)$ (Table 3). A moderate and positive correlation was observed between 6MWD and MIP ( $r=0.532, p=0.002)$, MEP ( $r=0.522$, $p=0.003)$, and shoulder flexion $(r=0.479, p=0.007)$. Knee extension strength $(r=0.399, p=0.029)$, shoulder abduction strength $(r=0.369, p=0.045)$, MELD score $(r=-0.404, p=0.027)$ also showed a weak correlation related to 6MWD. Furthermore, participants' physical status based on ASA classification was negatively and moderately related to 6MWD $(r=-0.404, p=0.027)$.

A multivariate regression analysis was performed to identify clinical parameters independently associated with 6MWD; age, MELD, MIP, MEP, knee extension, shoulder flexion, and shoulder abduction were included as clinical factors known to influence exercise capacity potentially. The obtained independent significant predictors of the 6MWD were age and maximal expiratory pressure; value accounted for $57.4 \%$ of the variance (Table 4 ). The MEP explains $14.3 \%$ of the variance as a relatively small proportion compared with age, explaining $46 \%$. These findings indicate the following equation: $6 \mathrm{MWD}(\mathrm{m})=540.05-(5.32 \times$ Age $)+(1.38 \times \mathrm{MEP})$

\section{DISCUSSION}

The present study assessed the relationship between liver disease severity, PA level, respiratory and skeletal muscle strength, fatigue, exercise capacity, physical performance, and HRQoL and determined the exercise capacity predictors in LT candidates. Based on the obtained results, we found that age and maximal expiratory pressure were the independent predictors of functional exercise capacity, and they explained $57.4 \%$ of the variance. The variables obtained from the univariate analysis significantly related to 6MWD were age, disease severity, ASA classification, respiratory and peripheral muscle strength, dyspnea perception before the 6-min walk test, and chair stands. However, no significant 
Table 2: Clinical Assessments of Liver Transplant Candidates.

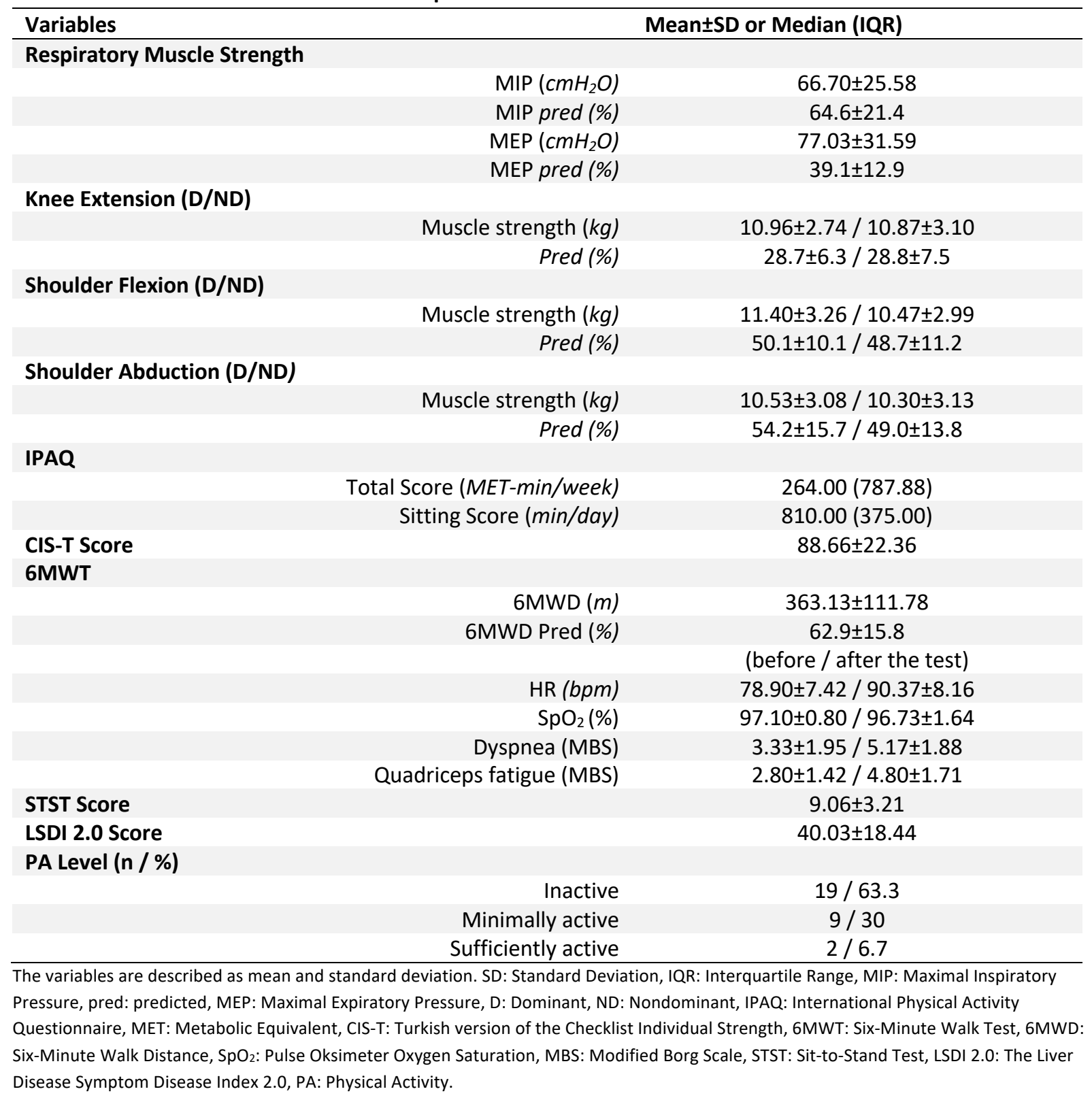

correlation was observed between exercise capacity and PA level, fatigue perception, and health-related quality of life. Our results highlight individual clinical characteristics' contribution in predicting those candidates unlikely to exhibit improvements in exercise capacity following LT surgery.

6MWT has been shown to be a reliable, functional measure used to evaluate pre-liver transplant exercise capacity (17) and predict morbidity and mortality $(11,18,19)$. Cirrhotic patients' survival analysis showed that individuals whose 6MWD are
$<410 \mathrm{~m}$ had a survival rate of $55 \%$ compared with $97 \%$ for individuals whose $>410 \mathrm{~m}$ (20). In our study, the maximum distance walked in 6-minutes $(363.13 \pm 111.78 \mathrm{~m})$ was found to be lower than healthy references $(570.47 \pm 86.68 \mathrm{~m})$, which is similar to the results reported by Carey et al. in patients with ESLD (369 m) (18). They also suggested that a decreased 6MWD might be considered another risk factor in conjunction with the MELD scoring, where patients have a high pre-transplant risk of death. In agreement with these studies, the 6MWD obtained in the present study corresponds to a high mortality risk; 
Table 3: Correlations Between 6MWD and Assessed Clinical Variables.

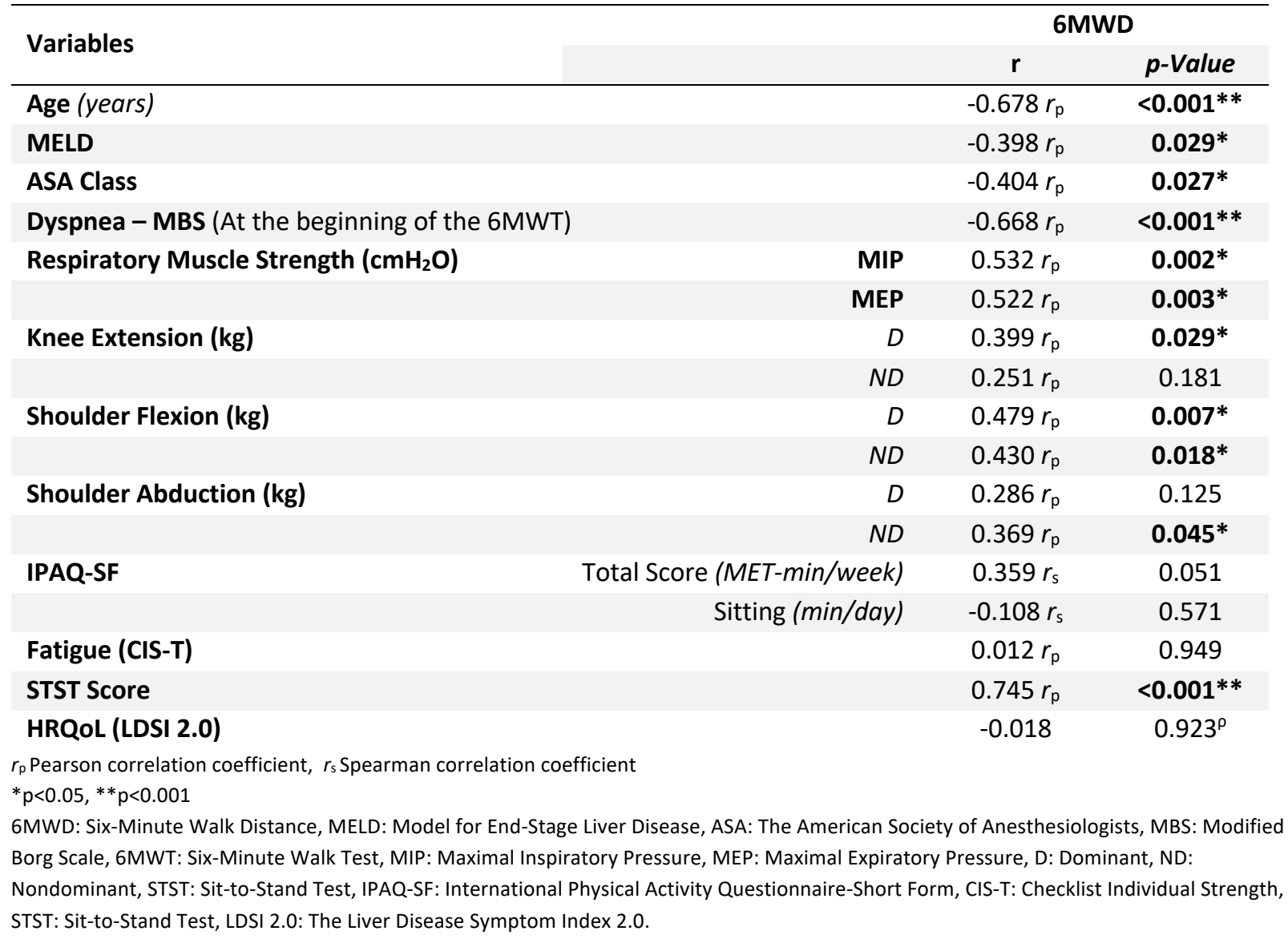

it is also negatively impacted by liver disease severity. Previous studies supported this finding, reporting wait-list patient with impaired walking distances to have significantly higher MELD scores than those who preserved 6MWD (19). In another study, MELD severity scores were shown to have an inverse correlation with respiratory musculature (21). It is of interest to note that the relationship between MELD scores and functional outcomes (obtained by physical performance test and 6MWT) was found to be weak in the present study. Here, the possible explanation could be that $37 \%$ of the patients had relatively low MELD scores (s15). Furthermore, Galant et al. found that having ascites in LT candidates contributed to the inverse correlation between MELD scores and 6MWD (2). Consistent with this finding, $46.7 \%$ of the participants had no ascites in the present study; it could also clarify the weak relation between the disease severity and exercise capacity. Additionally, subjects classified in higher ASA physical status had weaker shoulder abduction strength, MIP, MEP, and poor functional level obtained by 6MWD and
STSTwith a moderate correlation - independent of liver disease severity.

Older age is a challenging factor that impacts disease progression and advanced fibrosis and is independently associated with a higher mortality risk in the wait-list. It was revealed that age, calculated MELD score, and 6MWD were associated with survival in liver transplant candidates (19). Haugen et al. reported that older candidates were more likely to be frail, less likely to be robust, and had worse performance scores for all liver frailty index parameters; however, the impact of frailty did not vary by candidate age (22). One primary finding of the present study was older age, which identified as an independent predictor of low exercise capacity, causes lower walk distances and poor physical performance. Also, BMI was moderately and positively correlated with individuals' age.

Liver disease negatively impacts the musculature, including respiratory effects, evidenced by the high mortality rates with the lowest respiratory muscle 
Table 4: Multiple Linear Regression Analysis of Variables Independently Associated with 6MWD.

\begin{tabular}{lcccccc}
\hline $\begin{array}{l}\text { Independent } \\
\text { Variables }\end{array}$ & $\boldsymbol{R}$ & $\begin{array}{c}\text { Adjusted } \\
\boldsymbol{R}^{\mathbf{2}}\end{array}$ & $\begin{array}{c}\text { Regression } \\
\text { Coefficient (B) }\end{array}$ & $\begin{array}{c}\text { Standardized } \\
\text { Regression } \\
\text { Coefficient (Beta) }\end{array}$ & $\boldsymbol{t}$ & $\boldsymbol{P}$-Value \\
\hline (Constant) & 0.777 & 0.574 & 540.054 & & 7.101 & $<\mathbf{0 . 0 0 1}$ \\
Age & & & -5.321 & -0.591 & -4.750 & $<\mathbf{0 . 0 0 1}$ \\
MEP & & 1.376 & 0.389 & 3.126 & $\mathbf{0 . 0 0 4}$ \\
\hline
\end{tabular}

6MWD: Six-Minute Walk Distance; MEP: Maximal Expiratory Pressure

strength values; thus, breathing difficulties are the predominant presenting symptom in advanced liver diseases (20). Silva et al. reported that LT candidates had respiratory muscle weakness because of adynamia and the liver disease itself, and the preoperative muscle strength assessment could predict post-transplant pulmonary complications among this sample (23). The obtained results in the current study showed that respiratory muscle strength had a positively strong and/or moderate correlation with peripheral muscle strength and a moderate correlation with functional measure outcomes (chair stands and 6MWD). Our participants reported a moderate to severe dyspnea perception at the beginning of the 6MWT. On the other hand, concerning ascites contributing to respiratory difficulties, Kaltsakas et al. suggested that nonascites patients had better lung functions and significantly better MELD scores than ascites (3). Galant et al. also reported that higher MELD scores were associated with reduced inspiratory muscle strength (21). In the present study, ascites' prevalence was $53.3 \%$, with no correlation with any respiratory parameter.

Muscle wasting is well-recognized among cirrhosis related complications. It is suggested that myostatin might have a role in muscle wasting in ESLD, where these impairments appear to be independent of cirrhosis etiology (24). Wang et al. hypothesized that muscle function could serve as a comprehensive assessment of the musculoskeletal system and overall physical condition, global health status, consequently, most importantly, the patient's mobility and independence (25). These data confirm that our participants with higher muscle strength (shoulder flexion and knee extension) were more likely to show better STST scores, and the greater the distance walked the better the shoulder flexion strength. These findings suggest that although there is inadequate data on the relationship between liver cirrhosis and muscle strength, the identified reduction in exercise performance and muscle function requires measuring and improving wait-list individuals' muscle and exercise performance could potentially improve outcomes.

The age-related loss in muscle function is strongly related to poor physical performance tends to improve slowly; furthermore, it may decrease further in the post-liver transplant period (26). Lai et al. found that the average physical function of LT candidates worsened every 3 -months by $-0.38 \mathrm{~kg}$ in grip strength, $-0.05 \mathrm{~m} \mathrm{~s}^{-1}$ in gait, $0.03 \mathrm{sec}$ in chair testing, and -0.16 points in physical performance (4). This study revealed that the patients who had higher shoulder flexion, abduction, and knee extension strength showed better physical performances with a strong positive correlation. Participants with low physical performance scores had lower respiratory and peripheral muscle strength. Also, physical performance was inversely correlated with age, the presence of ascites, and MELD scores. In conjunction with these, it is emphasized that objective physical performance tests allow us to systematically identify cirrhotics for pre-rehabilitation interventions particularly where the timing is elective. It is believed that an attempt to increase physical fitness before orthotopic LT by target-specific training seems to be reasonable (27). Patients with cirrhosis waiting for transplantation are highly sedentary, and the selfreported PA levels in advanced liver disease subjects were reported as low, with all negative consequences in QoL aspects (28). Consistent with this, our sample with a high sedentary rate of $63.3 \%$ had poorer physical performances; interestingly, the correlation was determined as weak. However, self-assessment and provider-assessment could differ in indicating the actual performance of PA. (27). Individualized and 
standardized PA programs might be necessary to increase daily activity participation in patients awaiting LT (29).

This study has strengths and limitations. First, the present study is valuable in investigating the determinants of exercise capacity in liver transplant candidates, considering previous studies showed functional capacity as a predictor of morbidity and mortality in advanced liver patients. Otherwise, the study might have been limited using some subjective assessment methods and a relatively small sample size. Also, muscle status could not be evaluated using a muscle mass or volume measure.

In conclusion, assessing respiratory parameters might be critical in predicting exercise capacity, especially in older LT candidates. The prediction of individual functional risk parameters pre-operatively can be a guide in determining strategies to influence post-operative outcomes positively. Further studies on larger samples are needed to support our findings. The study also continues as an ongoing research to obtain liver recipients' post-transplant functional outcomes.

Acknowledgments: The study was performed at Memorial Ataşehir Hospital, İstanbul, Turkey. The authors would like to thank Kâmil Yalçın Polat, MD, Prof, Head of the Department of Organ Transplantation Centre, Memorial Ataşehir Hospital, for enabling this study. The authors would also like to express their sincere gratitude for the material support to Feryal Subaşı, PT, Prof, Head of the Physiotherapy and Rehabilitation Department, Yeditepe University.

Conflict of Interest: The authors state no conflict of interest.

Financial Disclosure: The present study needed no financial support, and the funders had no role in the design, conduct, or reporting of the research.

Ethical Approval: This study has been approved by the Ethics Committee of Non-Interventional Research in Dokuz Eylül University (date: 07.09 .2017 ; no: 2017/21-61) and adhered to the Declaration of Helsinki.

Informed Consent: All participants have signed a fully informed written consent form.

Peer-review: Externally peer-reviewed.

\section{REFERENCES}

1. Asrani SK, Devarbhavi H, Eaton J, Kamath PS. Burden of liver diseases in the world. J Hepatology 2019;70(1):151-171.

2. Galant L, Ferrari R, Forgiarini Jr L, Monteiro M, Marroni C, Dias A. Relationship between MELD severity score and the distance walked and respiratory muscle strength in candidates for liver transplantation. Transplant Proc 2010;42(5):17291730.
3. Kaltsakas G, Antoniou E, Palamidas AF, et al. Dyspnea and respiratory muscle strength in endstage liver disease. World J Hepatol 2013;5(2):56.

4. Lai JC, Dodge JL, Sen S, Covinsky K, Feng S. Functional decline in patients with cirrhosis awaiting liver transplantation: Results from the functional assessment in liver transplantation (FrAILT) study. Hepatology 2016;63(2):574-580.

5. Jurado R, Morales I, Taboada D, et al. Coping strategies and quality of life among liver transplantation candidates. Psicothema 2011;23(1):74-79.

6. Garcia A, Veneroso C, Soares D, Lima A, Correia $M$. Effect of a physical exercise program on the functional capacity of liver transplant patients. Transplant Proc 2014;46(6):1807-1808.

7. Saglam M, Arikan H, Savci S, et al. International physical activity questionnaire: reliability and validity of the Turkish version. Percept Mot Skills 2010;111(1):278-284.

8. Black LF, Hyatt RE. Maximal respiratory pressures: normal values and relationship to age and sex. Am J Respir Crit Care Med 1969;99(5):696-702.

9. Bohannon RW. Muscle strength: clinical and prognostic value of hand-grip dynamometry. Curr Opin Clin Nutr Metab Care 2015;18(5):465-470.

10. Ergin G, Yildirim Y. A validity and reliability study of the Turkish Checklist Individual Strength (CIS) questionnaire in musculoskeletal physical therapy patients. Physiother Theory Pract 2012;28(8):624632.

11.VanWagner LB, Uttal S, Lapin B, et al. Use of SixMinute Walk Test to Measure Functional Capacity After Liver Transplantation. Phys Ther 2016;96(9):1456-1467.

12. Taşkın B, Vardar-Yagli N, Calik-Kutukcu E, et al. An Evaluation Functional Capacity in Relation to Dyspnea and Fatigue, Muscle Strength, and Dynamic Balance in Patients with Liver Transplantation. Eur Respir J 2019;54(Suppl):63, PA1211.

13.Enright PL, Sherrill DL. Reference equations for the six-minute walk in healthy adults. Am J Respir Crit Care Med 1998;158(5):1384-1387.

14. Zanini A, Aiello M, Cherubino $F$, et al. The one repetition maximum test and the sit-to-stand test in the assessment of a specific pulmonary rehabilitation program on peripheral muscle strength in COPD patients. Int J Chron Obstruct Pulmon Dis 2015;10:2423-2430. 
15.Eraydın A, Akarsu M, Hakim GD, Keskinoğlu P, Ellidokuz $\mathrm{H}$. The validity and reliability of "The liver disease symptom index 2.0 " for Turkish society. Turk J Gastroenterol 2014;25(05):531-538.

16.Pleeging $\mathrm{E}$, Burger $\mathrm{M}$, van Exel J. The relations between hope and subjective well-being: $A$ literature overview and empirical analysis. Appl Res Qual Life 2019;1-23.

17.Lai JC, Volk ML, Strasburg D, Alexander N. Performance-based measures associate with frailty in patients with end-stage liver disease. Transplantation 2016;100(12):2656-2660.

18.Carey EJ, Steidley DE, Aqel BA, et al. Six-minute walk distance predicts mortality in liver transplant candidates. Liver Transpl 2010;16(12):1373-1378.

19. Yadav A, Chang YH, Carpenter $S$, et al. Relationship between sarcopenia, six-minute walk distance and health-related quality of life in liver transplant candidates. Clin Transpl 2015;29(2):134-141.

20.Faustini Pereira JL, Galant LH, Rossi D, et al. Functional capacity, respiratory muscle strength, and oxygen consumption predict mortality in patients with cirrhosis. Can J Gastroenterol Hepatol 2016;2016, Article ID 6940374.

21.Galant LH, Junior LAF, Dias AS, Marroni CA. Functional status, respiratory muscle strength, and quality of life in patients with cirrhosis. Rev Bras Fisioter 2012;16(1):30-34.

22. Haugen CE, McAdams-DeMarco M, Holscher CM, et al. Multicenter study of age, frailty, and waitlist mortality among liver transplant candidates. Ann Surg 2020;271(6):1132-1136.

23.da Silva ÁM, Cliquet Jr A, Boin I. Profile of respiratory evaluation through surface electromyography, manovacuometry, and espirometry in candidates on the liver transplant waiting list. Transplant Proc 2012;44(8):24032405.

24.Dasarathy S. Etiology and management of muscle wasting in chronic liver disease. Curr Opin Gastroenterol 2016;32(3):159-165.

25.Wang CW, Feng S, Covinsky KE, et al. A comparison of muscle function, mass, and quality in liver transplant candidates: results from the functional assessment in liver transplantation (FrAILT) study. Transplantation 2016;100(8):1692-1698.

26.Dunn MA, Rogal SS, Duarte-Rojo A, Lai JC. Physical Function, Physical Activity, and Quality of
Life after Liver Transplantation. Liver Transpl 2020;26(5):702-708.

27.Dunn MA, Josbeno DA, Schmotzer AR, et al. The gap between clinically assessed physical performance and objective physical activity in liver transplant candidates. Liver Transpl 2016;22(10):1324-1332.

28.Ney M, Gramlich L, Mathiesen V, et al. Patientperceived barriers to lifestyle interventions in cirrhosis. Saudi J Gastroenterol 2017;23(2):97104.

29.Ergene T, Karadibak D, Polat KY. Fatigue and Physiotherapy in Liver Transplant Recipients. Clin Exp Health Sci 2019;9(3):278-282. 